\title{
Multistage Estimators for the Distributed Drive Articulated Steering Vehicle
}

\author{
Guangzong Gao, ${ }^{1}$ Jixin Wang $\mathbb{D},{ }^{1}$ Tao $\mathrm{Ma}^{2}$ Wenzhong Liu, ${ }^{2}$ and Tianlong Lei ${ }^{1}$ \\ ${ }^{1}$ School of Mechanical and Aerospace Engineering, Jilin University, Changchun 130025, China \\ ${ }^{2}$ State Key Laboratory of Smart Manufacturing for Special Vehicles and Transmission System, Baotou 014030, China \\ Correspondence should be addressed to Jixin Wang; jxwang@jlu.edu.cn
}

Received 6 June 2020; Accepted 15 September 2020; Published 6 October 2020

Academic Editor: Luigi Rodino

Copyright (C) 2020 Guangzong Gao et al. This is an open access article distributed under the Creative Commons Attribution License, which permits unrestricted use, distribution, and reproduction in any medium, provided the original work is properly cited.

\begin{abstract}
The distributed drive articulated steering vehicle (DDASV) has a broad application prospect in the field of special operations. It is essential to obtain accurate vehicle states for better effect of active control. DDASV dynamic model is presented. To improve robustness, an adaptive strong tracking algorithm is applied to the singular value decomposition unscented Kalman filter (SVDUKF). Divided by yaw rate sensors and the tire models, two multistage estimators are established for DDASVs. Stable steering condition is simulated to investigate the influence on the estimated accuracy about the sensors and tire models. The velocities and tire forces are the key parameters to be estimated. The performance of each estimator regarding the practicability and accuracy is compared. The results show that all estimators are practicable. However, the accuracy of the estimated velocities based on yaw rate sensors is better and the transient tire model can improve the accuracy of estimated lateral forces more effectively for the estimator established with yaw rate sensors.
\end{abstract}

\section{Introduction}

The distributed drive articulated steering vehicle (DDASV) is a kind of articulated steering vehicle, which can realize active control and energy efficiency. It has a bright prospect on mine haulage, construction, and agriculture machinery $[1,2]$. Accurate state estimation of the key parameters like velocities and tire forces is more beneficial for vehicle control [3]. DDASVs have a smaller turning radius and better flexibility, and state estimation of DDASVs can improve the manipulation property, especially on the steering condition.

It is mature for passenger vehicles and trailers to estimate state values such as velocities, yaw rate, and tire forces $[4,5]$. Electronic stability program (ESP) sensors (yaw rate, longitudinal acceleration and lateral acceleration, wheel speeds) are the key to get the measurement parameters. An improved adaptive unscented Kalman filter was developed to estimate the longitudinal and lateral velocities with ESP sensors [6]. In references $[7,8]$, the tire forces were estimated with the measurement vector combined with ESP sensors. In reference [9], the tire forces are estimated with the measurement of yaw rate, longitudinal acceleration, and lateral acceleration only, with a difficulty that the observer gains were necessary but inaccessible. The lateral tire forces were estimated by neural network based on the simulated data from IPG CarMaker in reference [10]. It could realize the estimation without sensors but need more rigorous experimental data. For state estimation of articulated vehicles, the studies are focused on the free articulated vehicle like a tractor with a trailer. The sideslip based on Cheng and Cebon's linear single-track 5-DOF yawroll model was estimated in reference [11]. The lateral velocity and yaw rate for articulated heavy vehicle were estimated in reference [12].

Kalman filter (KF) is taken as a basis. The KF obtains the feedback gain to correct the forecast error. For the nonlinear vehicle model, unscented Kalman filter (UKF) can develop the feasibility. UKF approximates the probability density distribution of nonlinear function by sampling method which realizes Bayesian recursion. 
There exist two potential problems for UKF. One is for the error covariance matrix, which may be negative on Cholesky decomposition. Here are some methods for this problem such as SR decomposition [13], singular value decomposition (SVD) [14], and adaptive noise variance [15]. SVD is more robust than SR decomposition and less complex than the adaptive noise variance method, so it is adopted in this study [16]. The other problem is to restrain the divergence of the error covariance matrix and improve the accuracy. Two methods are presented for this problem. One is taking the strong tracking filter, like the freezing $K(k)$ method and the $S$ method $[17,18]$. The other is taking the adaptive unscented Kalman filter (AUKF) [19], which takes more computing resources. Strong tracking filter methods need less CPU resources, but the optimal tracking coefficient is difficult to be confirmed.

An adaptive strong tracking filter is applied for singular value decomposition unscented Kalman filter (SVDUKF). The SVDUKF overcomes the disadvantages of traditional AUKF and strong tracking UKF.

An 8-DOF DDASV model is presented for state estimation on the steering condition. The adaptive strong tracking SVDUKF is taken as the filter algorithm. Based on the vehicle dynamic model and the filter algorithm, two multistage estimators are established to estimate the velocities and tire forces. Two multistage estimators divided by the yaw rate sensors and the tire models are presented to estimate the velocities and tire forces of the DDASV. The performances regarding the estimated accuracy are compared, and conclusions are presented in the end.

\section{Creating of Vehicle Dynamic Model}

2.1. The Vehicle Model. The dynamic model of the DDASV with longitudinal, lateral, yaw, and articulated steering motions is presented, as shown in Figure 1. In this model, the vertical, roll, and pitch motions are omitted. The driving of each wheel is independent.

The longitudinal motion is expressed as follows:

$$
\begin{aligned}
& a_{x}=\frac{1}{m} \sum_{j=1}^{2}\left[\left(F_{x j}-R_{x j}\right) \cos \delta-F_{y j} \sin \delta\right]+\frac{1}{m} \sum_{j=3}^{4}\left[\left(F_{y j}-R_{x j}\right)\right], \\
& a_{x}=\frac{m_{f}}{m}\left[\dot{v}_{x f}-v_{y f}\left(\varphi_{f}+\varphi\right)\right]+\frac{m_{r}}{m}\left[\dot{v}_{x r}-v_{y r}\left(\varphi_{r}+\varphi\right)\right] .
\end{aligned}
$$

The lateral motion is expressed as follows:

$$
\begin{aligned}
& a_{y}=\frac{1}{m} \sum_{j=1}^{2}\left[\left(F_{x j}-R_{x j}\right) \sin \delta+F_{y j} \cos \delta\right]+\frac{1}{m} \sum_{j=3}^{4}\left[\left(F_{y j}\right)\right], \\
& a_{y}=\frac{m_{f}}{m}\left[\dot{v}_{y f}+v_{x f}\left(\varphi_{f}+\varphi\right)\right]+\frac{m_{r}}{m}\left[\dot{v}_{y r}+v_{x r}\left(\varphi_{r}+\varphi\right)\right]
\end{aligned}
$$

According to the rigid body kinematics,

$$
\begin{aligned}
& \dot{v}_{x f}-v_{y f}\left(\varphi_{f}+\varphi\right)=\dot{v}_{x}-v_{y}\left(\varphi_{f}+\varphi\right)-\left(\dot{\varphi}_{f}+\dot{\varphi}\right) l_{m f} \sin \delta-\left(\varphi_{f}+\varphi\right)^{2} l_{m f} \cos \delta, \\
& \dot{v}_{x r}-v_{y r}\left(\varphi_{r}+\varphi\right)=\dot{v}_{x}-v_{y}\left(\varphi_{r}+\varphi\right)+\left(\varphi_{r}+\varphi\right)^{2} l_{m r}, \\
& \dot{v}_{y f}+v_{x f}\left(\varphi_{f}+\varphi\right)=\dot{v}_{y}+v_{x}\left(\varphi_{f}+\varphi\right)+\left(\dot{\varphi}_{f}+\dot{\varphi}\right) l_{m f} \cos \delta-\left(\varphi_{f}+\varphi\right)^{2} l_{m f} \sin \delta, \\
& \dot{v}_{y r}-v_{x r}\left(\varphi_{r}+\varphi\right)=\dot{v}_{y}+v_{x}\left(\varphi_{r}+\varphi\right)-\left(\varphi_{r}+\varphi\right)^{2} l_{m r} .
\end{aligned}
$$

The yaw dynamic motion is expressed as follows:

$$
\dot{\varphi}=\frac{1}{I_{z}}\left[\left(-F_{x 1}+R_{x 1}+F_{x 2}-R_{x 2}\right) \frac{B_{f}}{2}+\left(-F_{x_{3}}+R_{x 3}+F_{x 4}-R_{x 4}\right) \frac{B_{r}}{2}+\left(F_{y 1}+F_{y 2}\right) l_{f}-\left(F_{y 3}+F_{y 4}\right) l_{r}\right] .
$$

The articulate motion is expressed as

$$
\begin{aligned}
& \dot{\varphi}_{f}=\frac{1}{I_{z f}}\left[M+\left(F_{y 1}+F_{y 2}\right) l_{f}-\left(F_{x 1}-R_{x 1}\right) \frac{B_{f}}{2}+\left(F_{x 2}-R_{x 2}\right) \frac{B_{f}}{2}\right], \\
& \dot{\varphi}_{r}=\frac{1}{I_{z r}}\left[-M-\left(F_{y 3}+F_{y 4}\right) l_{r}-\left(F_{x_{3}}-R_{x 3}\right) \frac{B_{r}}{2}+\left(F_{x 4}-R_{x 4}\right) \frac{B_{r}}{2}\right] .
\end{aligned}
$$




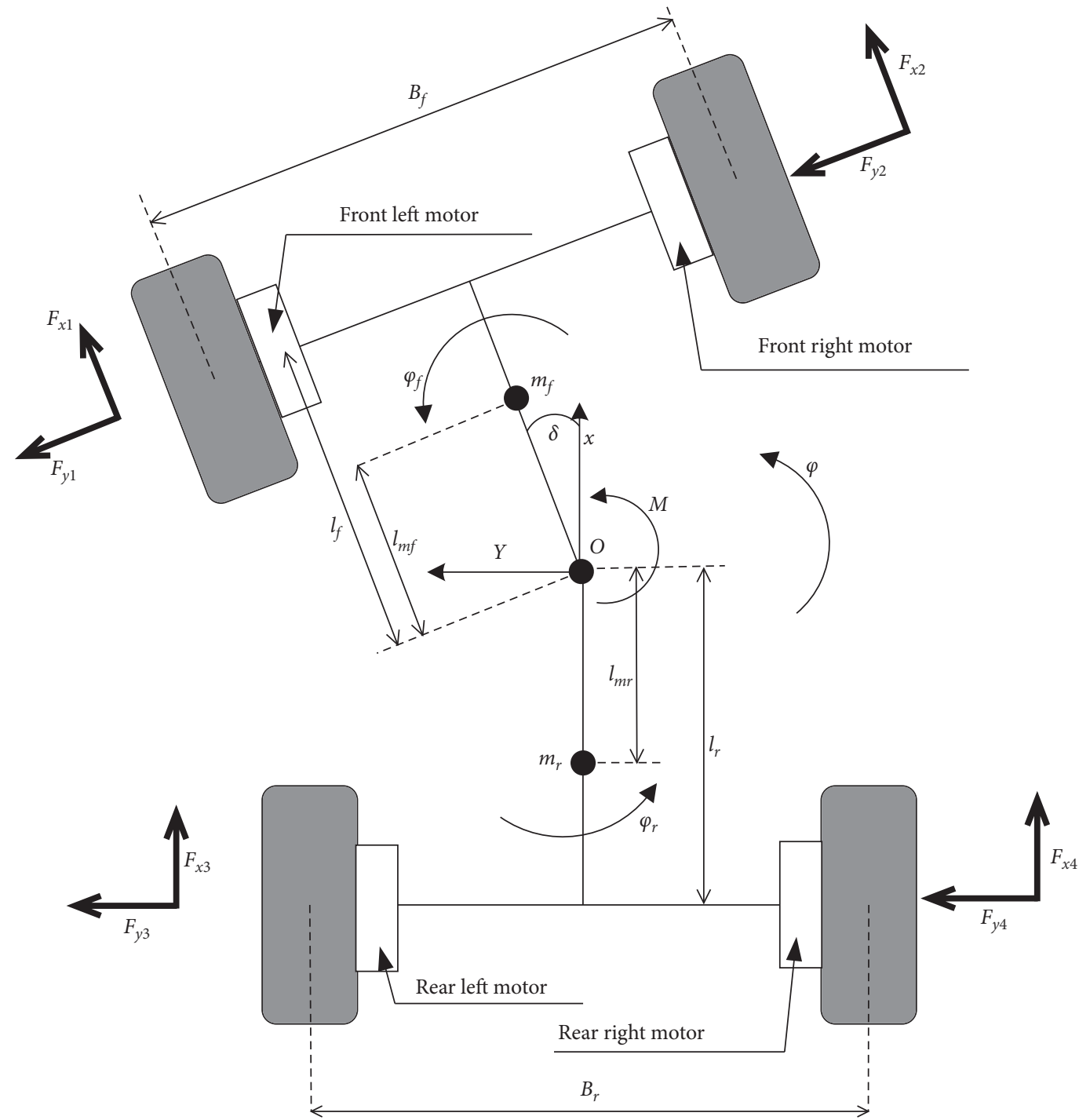

Figure 1: An 8-DOF DDASV model.

The vertical tire forces can be expressed as

$$
\begin{aligned}
& F_{z 1}=\frac{\left((1 / 2)-\left(\Delta l_{f}^{\prime} / l_{f}^{\prime}\right)\right)\left[(1 / 2)+\left(l_{f}-l_{m f}\right) \sin ((1 / 2) \delta)\right]}{\left(B_{f} \cos ((1 / 2) \delta)\right) m g}+\frac{h}{\left(B_{f} \cos ((1 / 2) \delta)\right) m a_{y}}+\left(\frac{1}{2}-\frac{\Delta l_{f}^{\prime}}{h}\right) m a_{x}, \\
& F_{z 2}=\frac{\left((1 / 2)+\left(\Delta l_{f}^{\prime} / l_{f}^{\prime}\right)\right)\left[(1 / 2)-\left(l_{f}-l_{m f}\right) \sin ((1 / 2) \delta)\right]}{\left(B_{f} \cos ((1 / 2) \delta)\right) m g}-\frac{h}{\left(B_{f} \cos ((1 / 2) \delta)\right) m a_{y}}+\left(\frac{1}{2}+\frac{\Delta l_{f}^{\prime}}{h}\right) m a_{x}, \\
& F_{z 3}=\frac{\left((1 / 2)-\left(\Delta l_{r}^{\prime} / l_{r}^{\prime}\right)\right)\left[(1 / 2)+\left(l_{r}-l_{m r}\right) \sin ((1 / 2) \delta)\right]}{\left(B_{r} \cos ((1 / 2) \delta)\right) m g}+\frac{h}{\left(B_{r} \cos ((1 / 2) \delta)\right) m a_{y}}-\left(\frac{1}{2}-\frac{\Delta l_{r}^{\prime}}{h}\right) m a_{x}, \\
& F_{z 4}=\frac{\left((1 / 2)+\left(\Delta l_{r}^{\prime} / l_{r}^{\prime}\right)\right)\left[(1 / 2)-\left(l_{r}-l_{m r}\right) \sin ((1 / 2) \delta)\right]}{\left(B_{r} \cos ((1 / 2) \delta)\right) m g}-\frac{h}{\left(B_{r} \cos ((1 / 2) \delta)\right) m a_{y}}-\left(\frac{1}{2}+\frac{\Delta l_{r}^{\prime}}{h}\right) m a_{x} .
\end{aligned}
$$


The assistant geometrical parameters $\Delta l_{f}^{\prime}, l_{f}^{\prime}, \Delta l_{r}^{\prime}, l_{r}^{\prime}$ are expressed as follows:

$$
\begin{aligned}
l_{f}^{\prime} & =l_{f} \cos \left(\frac{1}{2} \delta\right)+\frac{1}{2} B_{f} \sin \left(\frac{1}{2} \delta\right), \\
\Delta l_{f}^{\prime} & =\frac{1}{2} B_{f} \sin \left(\frac{1}{2} \delta\right), \\
l_{r}^{\prime} & =l_{r} \cos \left(\frac{1}{2} \delta\right)+\frac{1}{2} B_{r} \sin \left(\frac{1}{2} \delta\right), \\
\Delta l_{r}^{\prime} & =\frac{1}{2} B_{r} \sin \left(\frac{1}{2} \delta\right) .
\end{aligned}
$$

2.2. The Quasistatic and Transient Tire Models. The quasistatic tire model of Dugoff is adopted in this study. The longitudinal tire force $F_{x}$ and lateral tire force $F_{y}$ can be given as

$$
\begin{aligned}
& F_{x}=C_{\sigma} \frac{\sigma_{x}}{1+\sigma_{x}} f(\lambda), \\
& F_{y}=C_{\alpha} \frac{\tan (\alpha)}{1+\sigma_{x}} f(\lambda),
\end{aligned}
$$

where $\lambda$ is given as follows:

$$
\lambda=\frac{\mu F_{z}\left(1+\sigma_{x}\right)}{2\left[\left(C_{\sigma} \sigma_{x}\right)^{2}+\left(C_{\sigma} \tan (\alpha)\right)^{2}\right]^{1 / 2}},
$$

and $f(\lambda)$ is given as

$$
f(\lambda)= \begin{cases}(2-\lambda) \lambda, & \lambda<1, \\ 1, & \lambda \geq 1 .\end{cases}
$$

The transient tire model to calculate the lateral tire forces can be expressed as follows:

$$
\dot{F}_{y}=\frac{v}{\tau}\left(-F_{y}+\bar{F}_{y}\right)
$$

where $v$ denotes the longitudinal velocity of the wheel, $\tau$ denotes the relaxation coefficient, and $\bar{F}_{y}$ denotes the lateral tire force from the quasistatic tire model of Dugoff.

\section{Singular Value Decomposition Unscented Kalman Filter with Adaptive Strong Tracking}

3.1. Singular Value Decomposition Unscented Kalman Filter. Unlike the extended Kalman filter (EKF), the UKF approximates the probability density distribution of nonlinear functions by sampling, which realizes the Bayesian estimation too. This makes it imperative for UKF to sample and weight. With measurement error and system disturbance, the error covariance matrix might lose positive semidefinite, which makes Cholesky decomposition fail during sampling and weighting.

With no limit of positive definiteness of the decomposed matrix, the SVD has higher robustness. The process of SVDUKF can be expressed via the following equations.

The system state function and measurement function can be expressed as follows:

$$
\begin{aligned}
X(k+1) & =\varphi[X(k), W(k), k], \\
Z(k) & =h[X(k), V(k), k] .
\end{aligned}
$$

Initial conditions:

$$
\begin{aligned}
& \widehat{X}(0)=E[X(0)], \\
& P(0)=E\left\{[X(0)-\widehat{X}(0)][X(0)-\widehat{X}(0)]^{T}\right\} .
\end{aligned}
$$

Sampling and weighting based on SVD are expressed as equations (29)-(35).

The error covariance matrix $P$ can be expressed by the decomposition matrix $U, S$, and $V$ :

$$
P^{1 / 2}=U\left[\begin{array}{ll}
S & 0 \\
0 & 0
\end{array}\right] V^{T},
$$

$$
\begin{aligned}
x_{0}(k-1) & =\bar{X}(k-1), \\
x_{i}(k-1) & =\bar{X}(k-1)+\rho U_{i} \sqrt{s_{i}} \quad i=1, \ldots, n, \\
x_{i}(k-1) & =\bar{X}(k-1)-\rho U_{i-n} \sqrt{s_{i-n}}, \quad i=(n+1), \ldots, 2 n,
\end{aligned}
$$

where $\rho$ is the sigma coefficient. The weighting values $W_{0}^{m}$, $W_{0}^{p}, W_{i}^{m}, W_{i}^{p}$ can be expressed as follows, and $\lambda$ is a conversion coefficient, $\lambda=\alpha^{2}(n+\kappa)$ :

$$
\begin{aligned}
\rho & =\alpha \sqrt{n+\kappa} \\
W_{0}^{m} & =W_{0}^{p}=\frac{\lambda}{(n+\lambda)}, \\
W_{i}^{m} & =W_{i}^{p}=\frac{1}{2(n+\lambda)}, \quad i=1, \ldots, 2 n .
\end{aligned}
$$

For equation (29), $S=\operatorname{diag}\left(s_{1}, s_{2}, \ldots, s_{n}\right)$. Generally, the error covariance matrix is a symmetric matrix, which means $U=V$, and the matrix eigenvalue is $\left[s_{1}^{2}, s_{2}^{2}, \ldots, s_{n}^{2}\right]$. Therefore, the eigenvectors of the error covariance matrix can be substituted by $U U^{T}$. For Gaussian noise distribution, when the state variable is a single variable, $\kappa=2$. When the state variable is multivariable, $\kappa=3$.

The time update function can be expressed as follows: 


$$
\begin{aligned}
& x_{i}\left(\frac{k}{k-1}\right)=\varphi\left[x_{i}(k-1)\right], \quad i=0, \ldots, 2 n, \\
& \widehat{X}\left(\frac{k}{k-1}\right)=\sum_{i=0}^{2 n} W_{i}^{m} x_{i}\left(\frac{k}{k-1}\right), \\
& P\left(\frac{k}{k-1}\right)=\sum_{i=0}^{2 n} W_{i}^{P}\left[x_{i}\left(\frac{k}{k-1}\right)-\widehat{X}\left(\frac{k}{k-1}\right)\right]\left[x_{i}\left(\frac{k}{k-1}\right)-\widehat{X}\left(\frac{k}{k-1}\right)\right]^{T}+Q, \\
& \zeta_{i}\left(\frac{k}{k-1}\right)=h\left[x_{i}\left(\frac{k}{k-1}\right)\right], \quad i=0, \ldots, 2 n, \\
& \widehat{Z}\left(\frac{k}{k-1}\right)=\sum_{i=0}^{2 n} W_{i}^{m} \zeta_{i}\left(\frac{k}{k-1}\right) .
\end{aligned}
$$
follows:

The measurement update function can be expressed as

$$
\begin{aligned}
P_{Z Z} & =\sum_{i=0}^{2 n} W_{i}^{p}\left[\zeta_{i}\left(\frac{k}{k-1}\right)-\widehat{Z}\left(\frac{k}{k-1}\right)\right]\left[\zeta_{i}\left(\frac{k}{k-1}\right)-\widehat{Z}\left(\frac{k}{k-1}\right)\right]^{T}+R, \\
P_{X Z} & =\sum_{i=0}^{2 n} W_{i}^{p}\left[x_{i}\left(\frac{k}{k-1}\right)-\widehat{X}\left(\frac{k}{k-1}\right)\right]\left[\zeta_{i}\left(\frac{k}{k-1}\right)-\widehat{Z}\left(\frac{k}{k-1}\right)\right]^{T}, \\
K(k) & =P_{X Z} P_{Z Z}^{-1} \\
\widehat{X}\left(\frac{k}{k}\right) & =\widehat{X}\left(\frac{k}{k-1}\right)+K(k)\left[Z(k)-\widehat{Z}\left(\frac{k}{k-1}\right)\right], \\
P\left(\frac{k}{k}\right) & =P\left(\frac{k}{k-1}\right)-K(k) P_{Z Z} K(k)^{T} .
\end{aligned}
$$

3.2. Adaptive Strong Tracking Algorithm. Because of the nonlinear model and the deviation between the mathematical model and the physical vehicle model, the theoretical mean square error and the Kalman filter gain become smaller over time. When the new data are used to correct the previous step estimation for extrapolation, the added weight decreases; meanwhile, the weight of the previous data increases. This results in the increase of cumulative error, eventually leading to data saturation and divergence.

According to the closed circuit, the strong tracking algorithm takes the fading factor $\xi$ into the propagated covariance $P(k \mid k-1)$. The fading factor increases the proportion of measurement in state estimation and suppresses filter divergence.

The propagated covariance $P(k \mid k-1)$ based on the adaptive strong tracking algorithm is expressed as follows: Innovation $v(k)$ can be expressed as follows:

$$
v(k)=Z(k)-\widehat{Z}\left(\frac{k}{k-1}\right) .
$$

Covariance matrix correctional parameter $\xi(k)$ can be calculated as follows: 


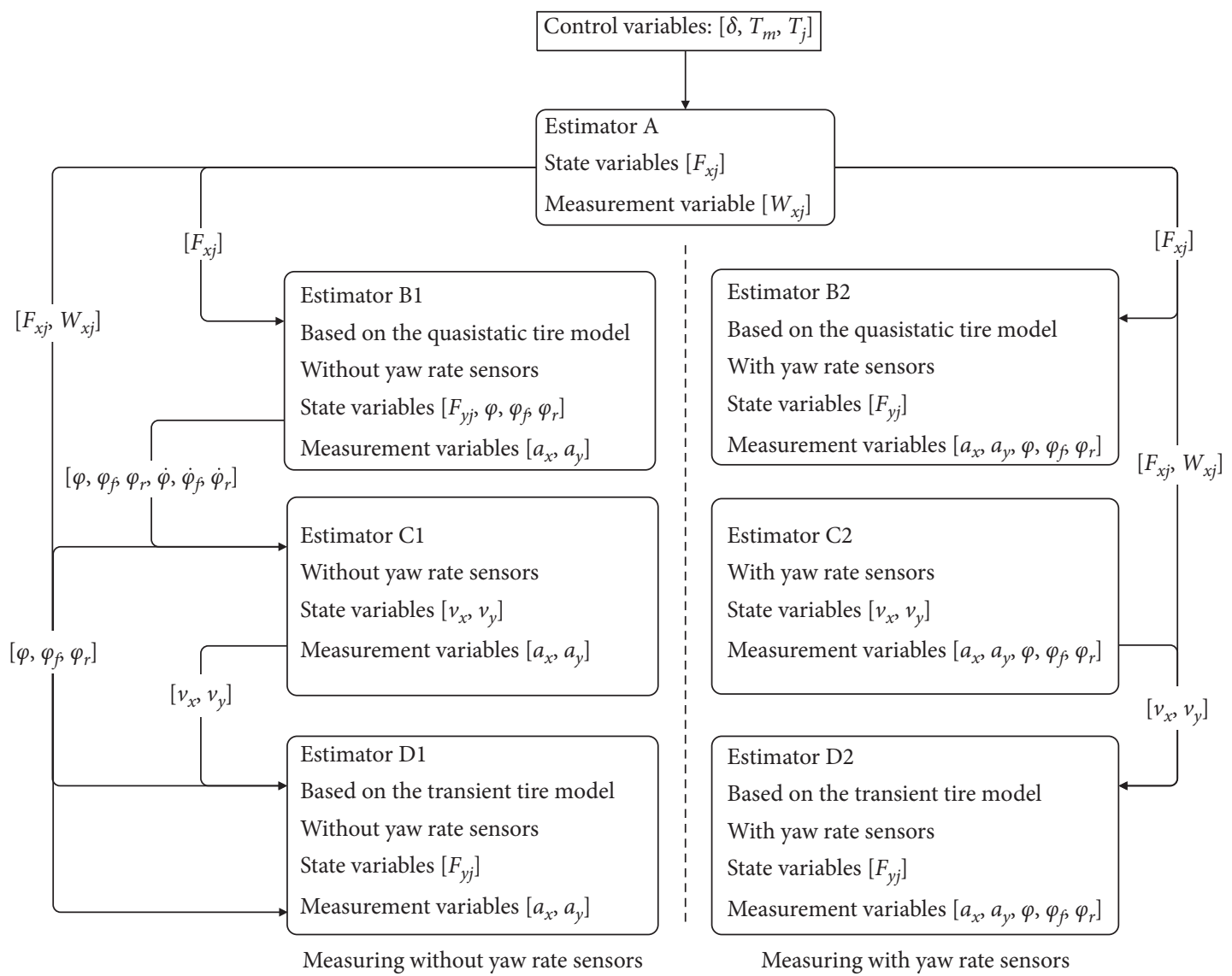

FIgURE 2: Block diagram of the comparison of multistage estimators.

$$
\text { if } \begin{aligned}
v^{T}(k) \cdot v(k) & \leq r \cdot \operatorname{tr}\left(P_{Z Z}\right), \xi(k)=1, \\
\text { else, } \xi(k) & =\frac{v(k) v^{T}(k)-Q^{\sim}-R}{P_{Z Z}-Q^{\sim}-R}, \\
P\left(\frac{k}{k-1}\right) & =\xi(k) \sum_{i=0}^{2 n} W_{i}^{P}\left[x_{i}\left(\frac{k}{k-1}\right)-\widehat{X}\left(\frac{k}{k-1}\right)\right]\left[x_{i}\left(\frac{k}{k-1}\right)-\widehat{X}\left(\frac{k}{k-1}\right)\right]^{T}+Q .
\end{aligned}
$$

For equation (47), $r$ is a reserve coefficient and $r \geq 1$. The covariance matrix of system noise $Q^{\sim}$ can be expressed as follows, where $q_{i}$ is the element of system noise matrix $Q$ :

$$
Q^{\sim}=\frac{1}{n} \sum_{i=1}^{n}\left[h\left(q_{i}\right)-h(\bar{Q})\right]\left[h\left(q_{i}\right)-h(\bar{Q})\right]^{T}
$$

3.3. Establishing Multistage Estimators. The multistage estimators are set as in Figure 2. The estimators B1, C1, and D1 are established without yaw rate sensors while the estimators B2, C2, and D2 are established with yaw rate sensors. The estimators B1 and B2 are established based on the quasistatic tire model while the estimators D1and D2 are established based on the transient tire model.

\section{Simulation Results and Analysis}

4.1. Initial Settings and Simulation Conditions. To evaluate the performance and compare the estimated accuracy of different estimators, computer simulation is implemented and the estimate algorithms are established. The process is shown in Figure 3. The noise is added at the road. The vehicle parameters are set as in Table 1.

The simulation condition is steering based on a stable longitudinal velocity at $3(\mathrm{~m} / \mathrm{s})$. The target and actual articulated steering angles are shown in Figure 4.

4.2. Results Analysis. The estimated results of the DDASV steering on the simulation condition can be shown as follows. Maximum absolute error (MAE) and root mean square error (RMSE) are adopted to compare the 


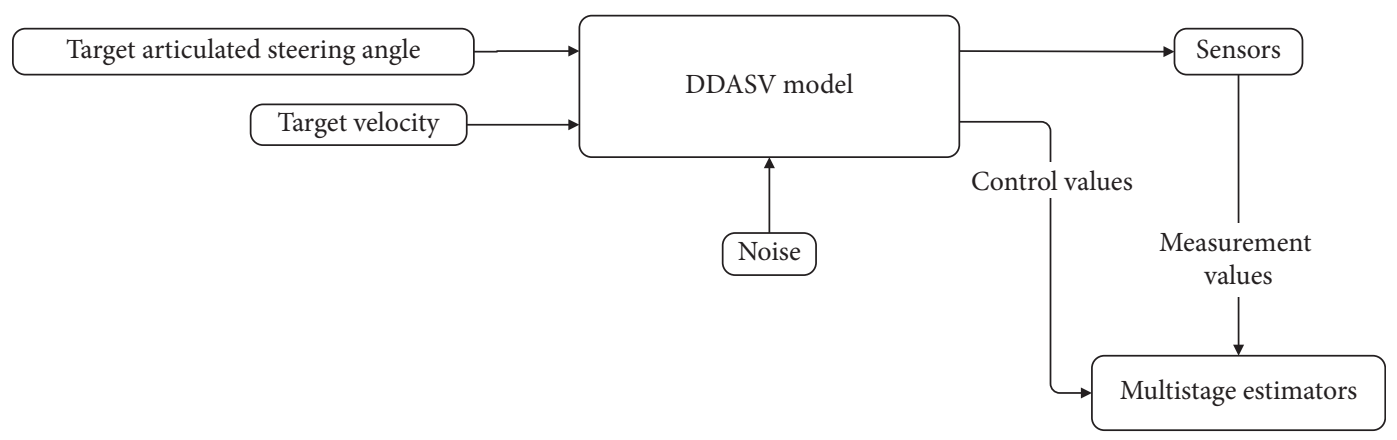

FIGURE 3: Block diagram of the simulation system.

TABLE 1: Parameters of the DDASV model.

\begin{tabular}{lcc}
\hline Parameter & Unit & \\
\hline Gross mass & $m(\mathrm{~kg})$ & Value \\
Mass of the front part vehicle & $m_{f}(\mathrm{~kg})$ & 2600 \\
Mass of the rear part vehicle & $m_{r}(\mathrm{~kg})$ & 1300 \\
Front wheel track & $B_{f}(\mathrm{~m})$ & 1300 \\
Rear wheel track & $B_{r}(\mathrm{~m})$ & 1.4 \\
Distance from articulated point to front axle & $l_{f}(\mathrm{~m})$ & 1.4 \\
Distance from articulated point to rear axle & $l_{r}(\mathrm{~m})$ & 1.2 \\
Distance from articulated point to the center of front vehicle gravity & $l_{m f}(\mathrm{~m})$ & 1.2 \\
Distance from articulated point to rear axle & $l_{m r}(\mathrm{~m})$ & 0.3 \\
Centroid height & $h(\mathrm{~m})$ & 0.3 \\
Wheel radius & $r(\mathrm{~m})$ & 0.4 \\
Vehicle rotational inertia about $z$-axis & $I_{z}\left(\mathrm{~kg} \times \mathrm{m}^{2}\right)$ \\
Front part vehicle rotational inertia about $z$-axis & $I_{z f}\left(\mathrm{~kg} \cdot \mathrm{m}^{2}\right)$ \\
Rear part vehicle rotational inertia about $z$-axis & $I_{z r}\left(\mathrm{~kg} \cdot \mathrm{m}^{2}\right)$ \\
\hline
\end{tabular}

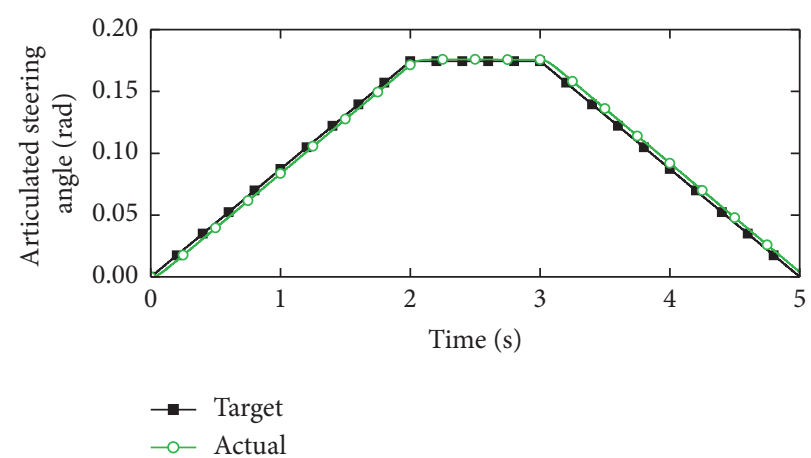

FIgURE 4: Target and actual articulated steering angles.

estimated accuracy of different estimators. Because of the zero-crossing point of the actual value, the mean absolute percentage error and the symmetric mean absolute percentage error cannot be applied to evaluate the practicability of different estimators. Evolution mean square percentage error (EMSPE) is taken as a replacement for mean absolute percentage error and the symmetric mean absolute percentage error. The EMSPE can be expressed as follows:
$\mathrm{EMSPE}=\sqrt{\frac{1}{n} \frac{\sum_{i=1}^{n}(\text { estimated value }- \text { actual value })^{2}}{\sum_{i=1}^{n}(\text { actual value })^{2}} 100 \%}$

As shown in Figure 5, the estimated errors of the longitudinal tire forces converge at about $0.15 \mathrm{~s}$. The estimated 


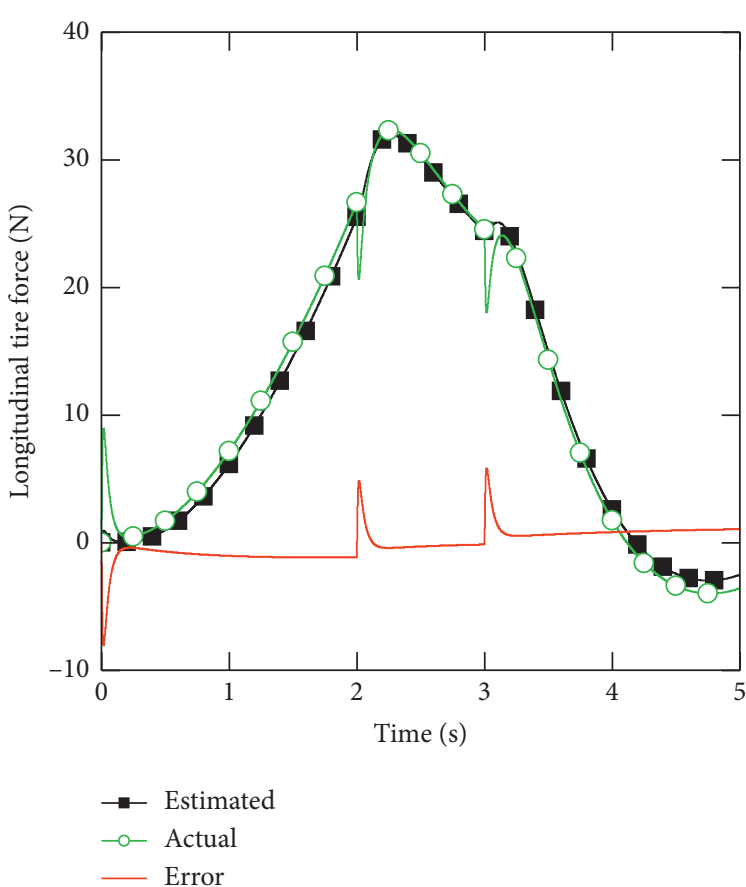

(a)

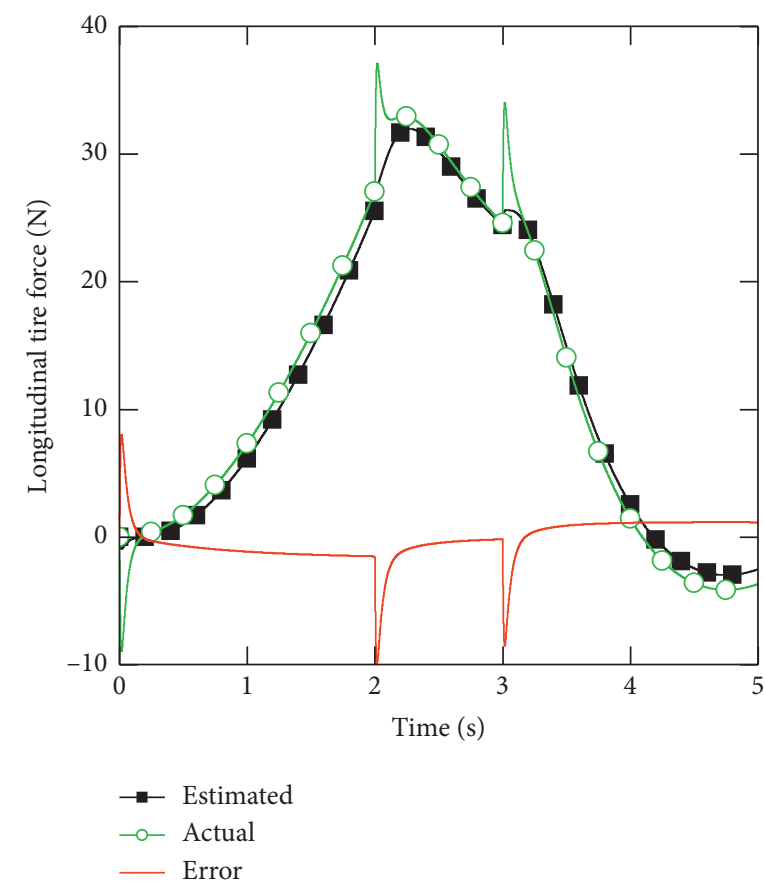

(c)

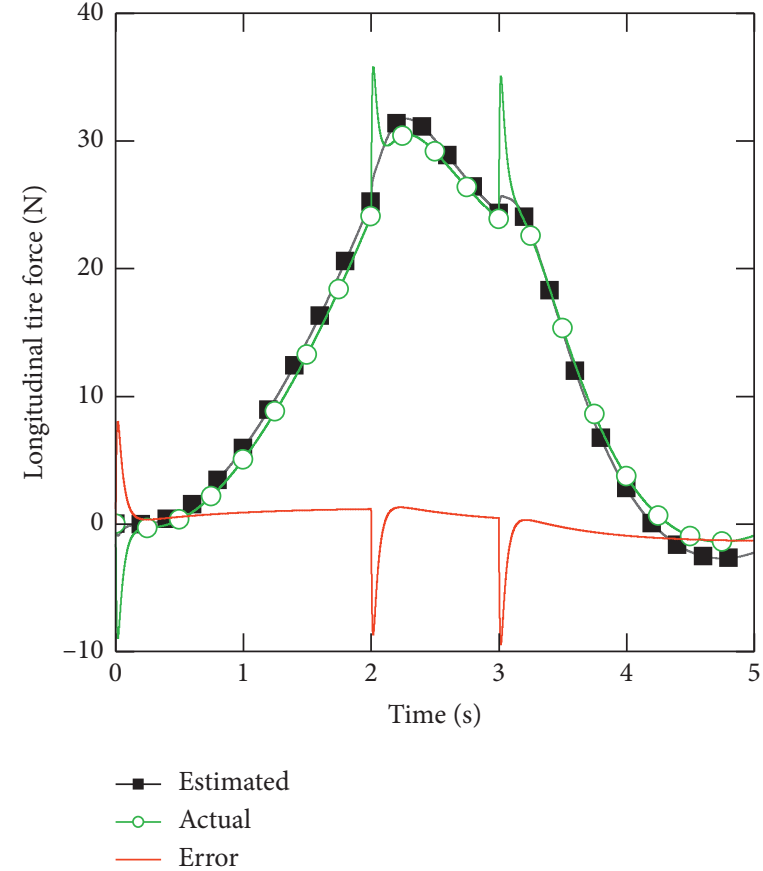

(b)

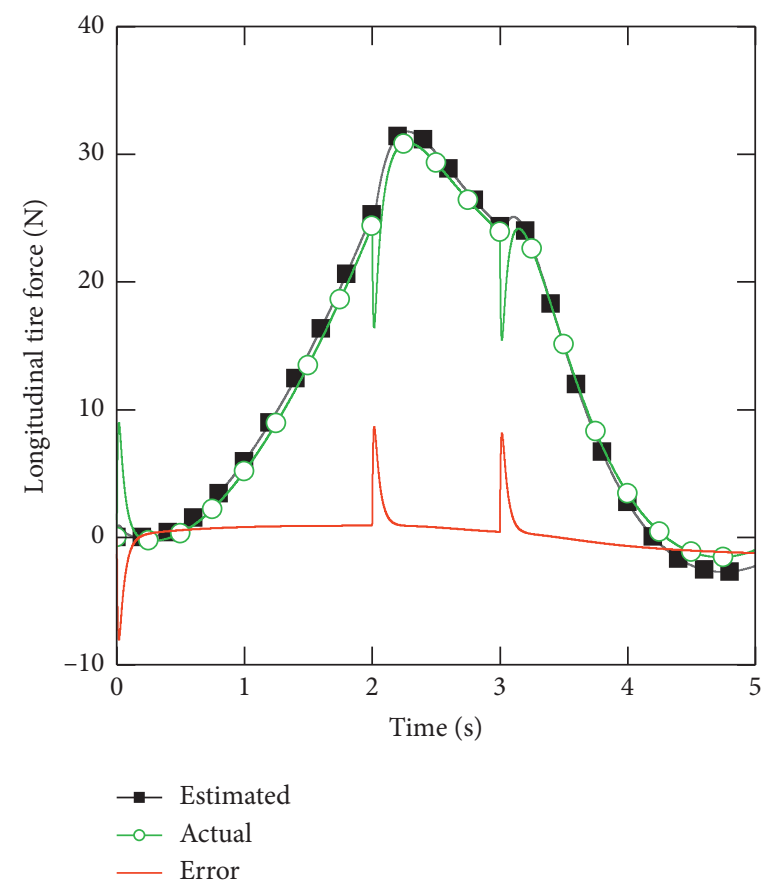

(d)

FIGURE 5: Estimated longitudinal tire forces based on the estimator A. (a) Front left longitudinal tire force. (b) Front right longitudinal tire force. (c) Rear left longitudinal tire force. (d) Rear right longitudinal tire force.

errors jump with the sudden of the actual values. The EMSPEs of the estimated longitudinal tire forces are $0.0336 \%, 0.0419 \%, 0.0435 \%$, and $0.0418 \%$, which, respectively, denote the results of the front left, front right, rear left, and rear right tires.

Figure 6 shows the estimated yaw rates based on the estimator B1. The EMSPEs of the estimated yaw rates are
$0.0063 \%, 0.0543 \%$, and $0.0507 \%$, which, respectively, denote the results of the yaw rate of the DDASV, articulated yaw rate of the front DDASV, and articulated yaw rate of the rear DDASV.

Figure 7 shows the estimated lateral tire forces based on the estimator B1. Figure 8 shows the estimated lateral tire forces based on the estimator B2. The EMSPEs of the 


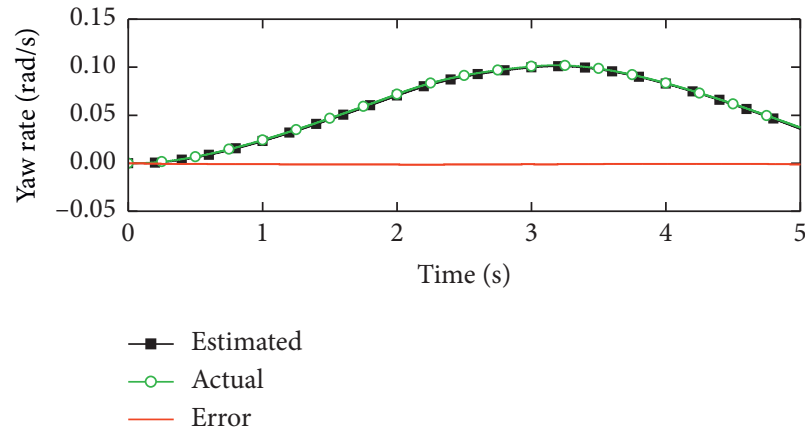

(a)

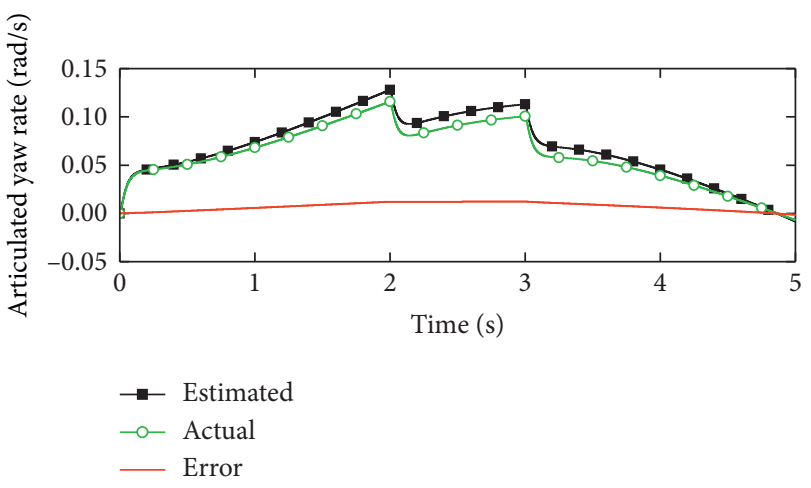

(b)

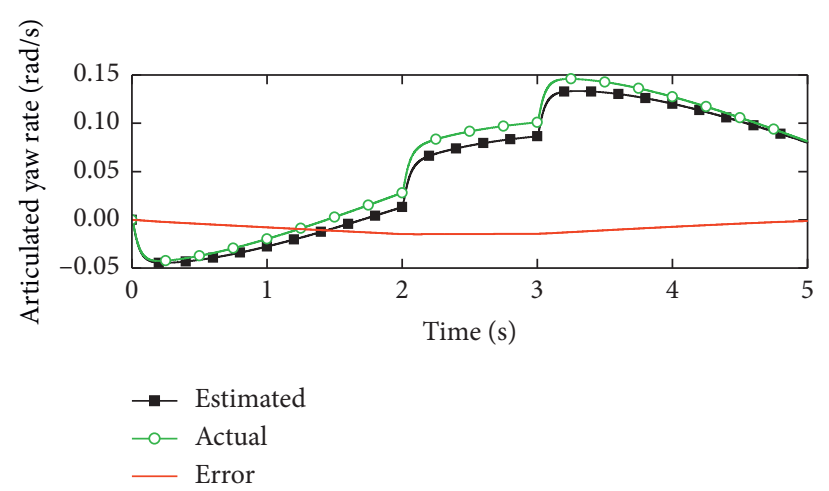

(c)

Figure 6: Estimated yaw rates based on the estimator B1 (estimator B1 is based on the quasistatic tire model without yaw rate sensors). (a) Yaw rate of the DDASV. (b) Articulated yaw rate of the front DDASV. (c) Articulated yaw rate of the rear DDASV.

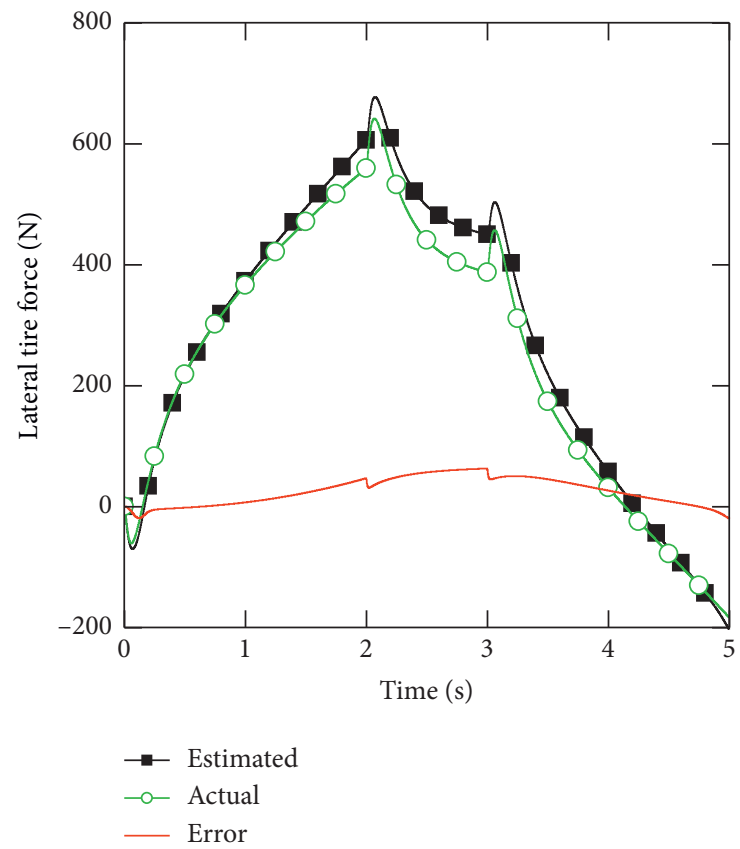

(a)

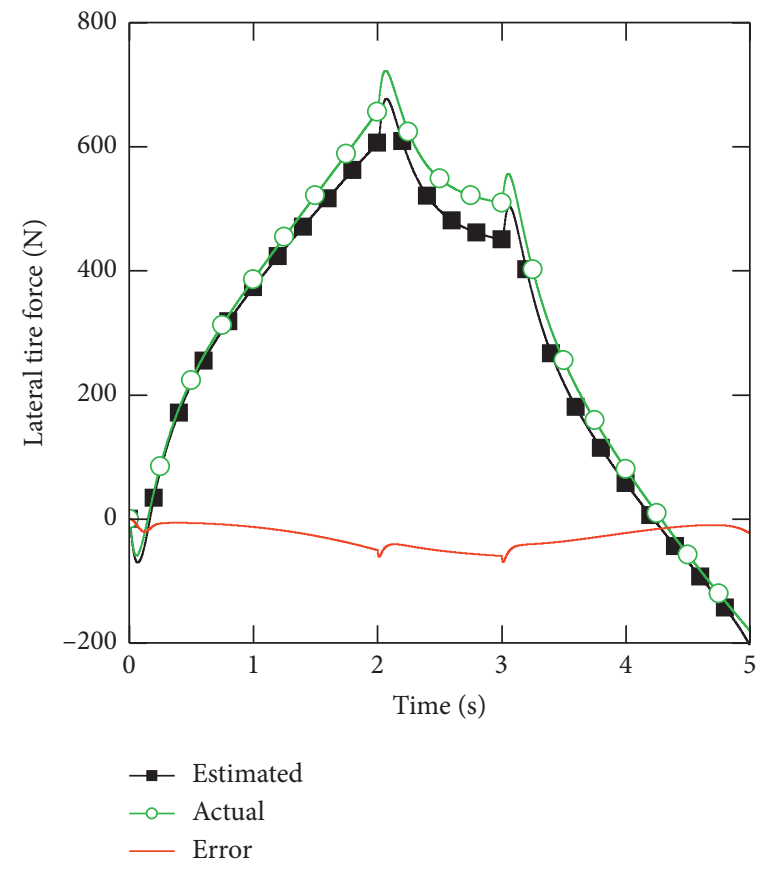

(b)

FIgURE 7: Continued. 


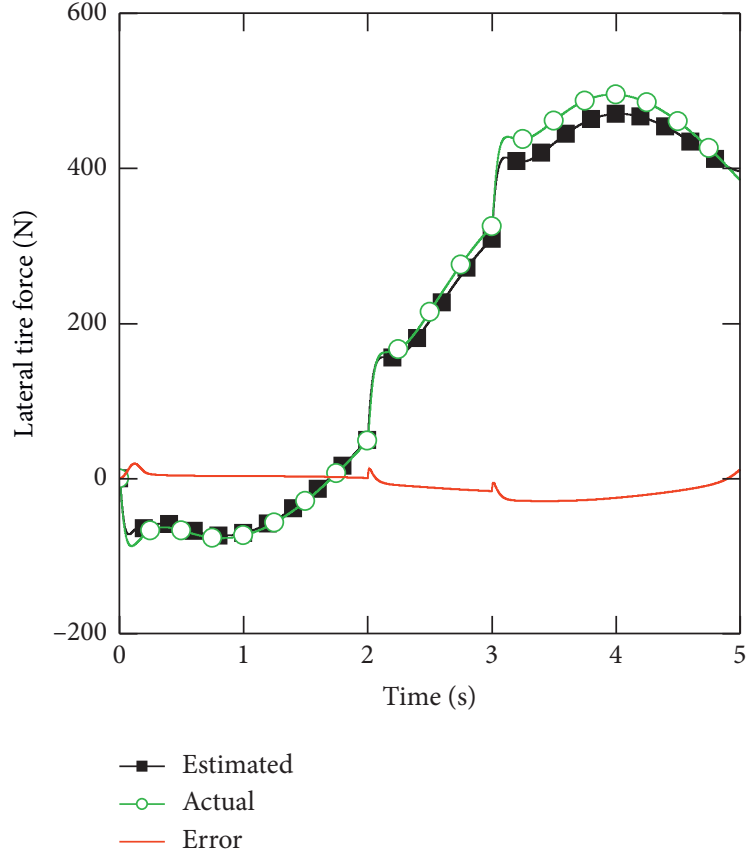

(c)

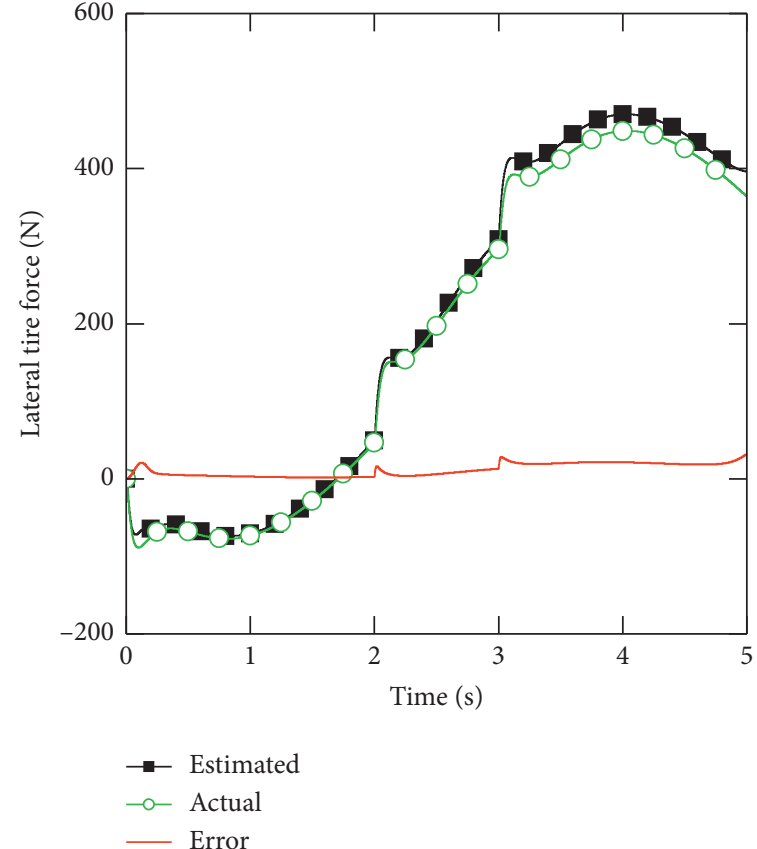

(d)

FiguRe 7: Estimated lateral tire forces based on the estimator B1 (estimator B1 is based on the quasistatic tire model without yaw rate sensors). (a) Front left lateral tire force. (b) Front right lateral tire force. (c) Rear left lateral tire force. (d) Rear right lateral tire force.

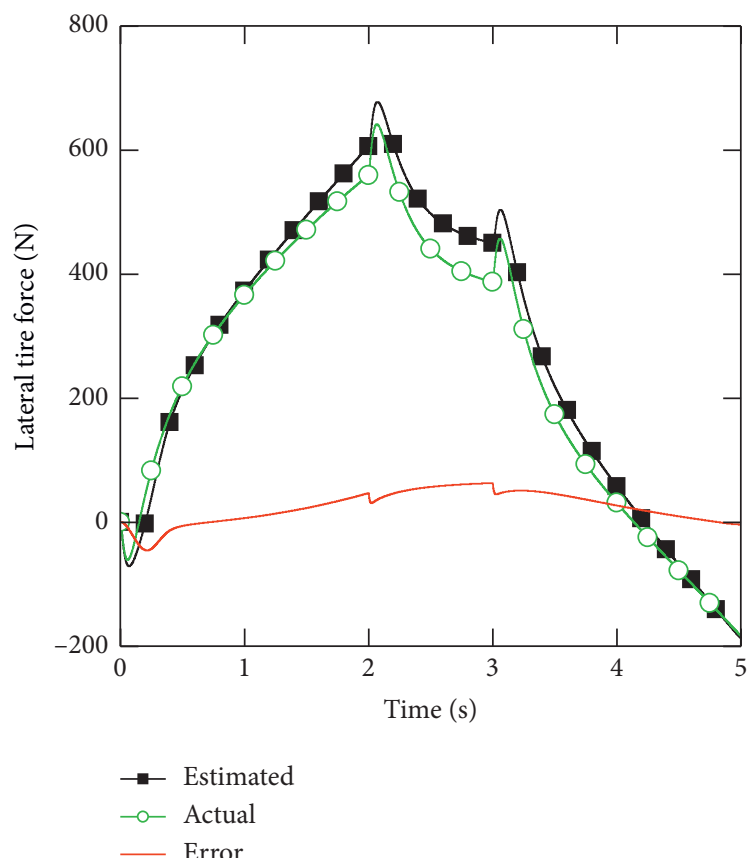

(a)

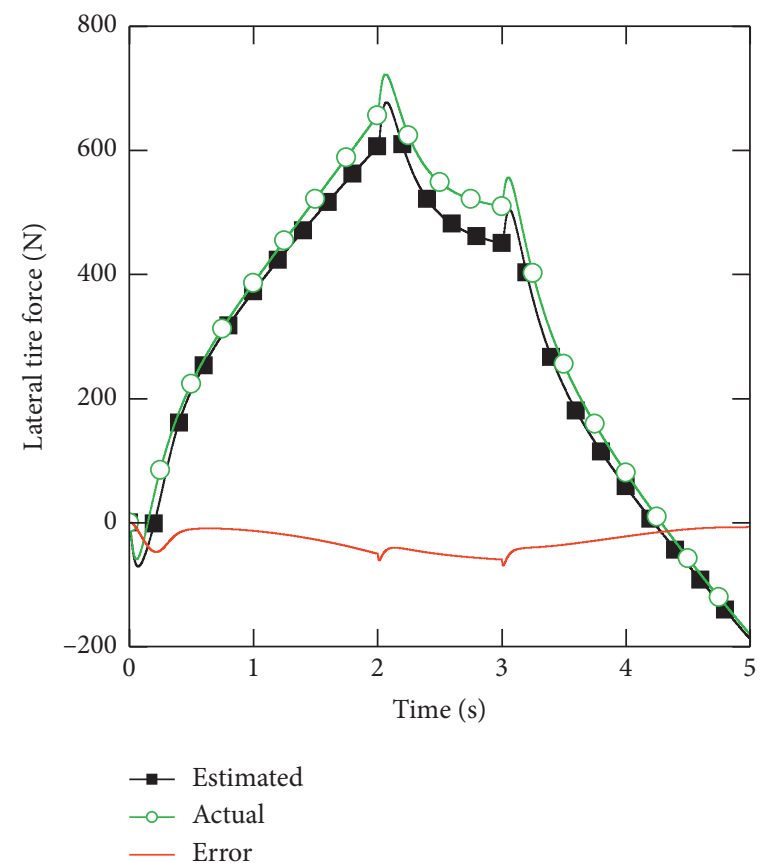

(b)

Figure 8: Continued. 


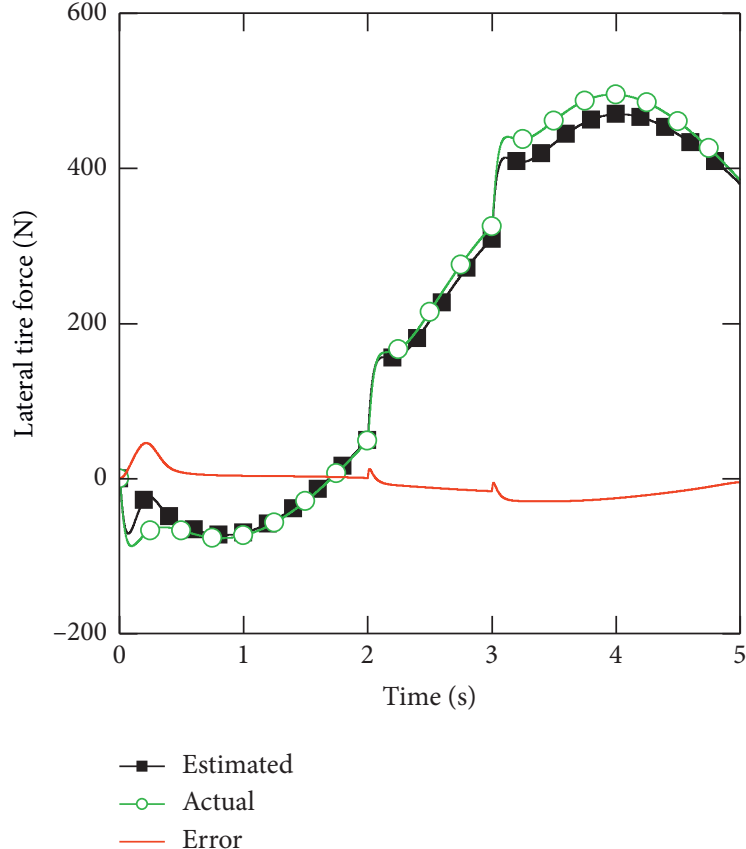

(c)

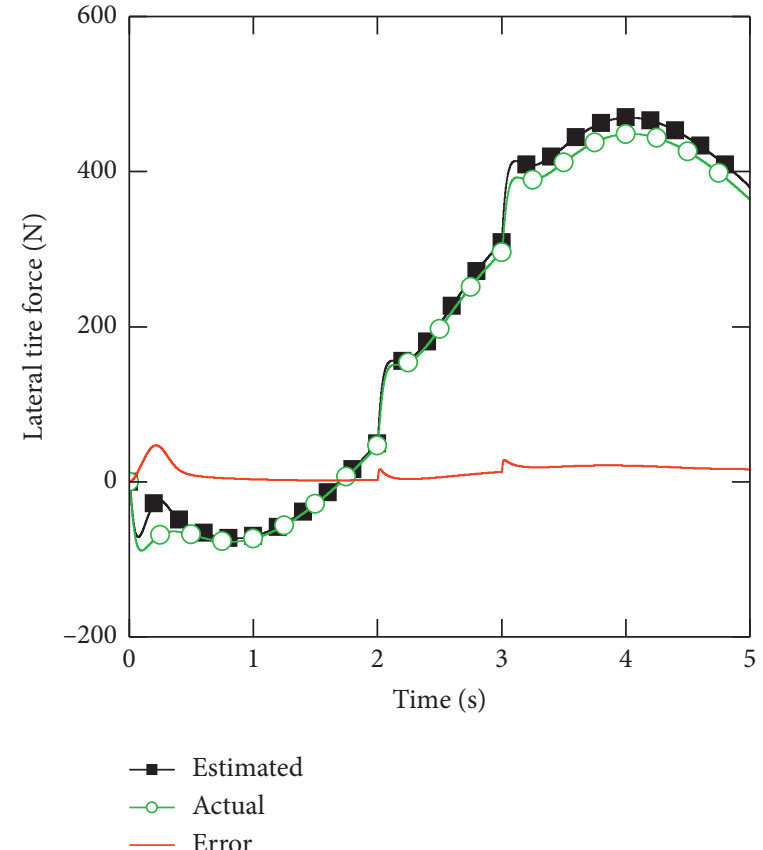

(d)

FIGURE 8: Estimated lateral tire forces based on the estimator B2 (estimator B2 is based on the quasistatic tire model with yaw rate sensors). (a) Front left lateral tire force. (b) Front right lateral tire force. (c) Rear left lateral tire force. (d) Rear right lateral tire force.

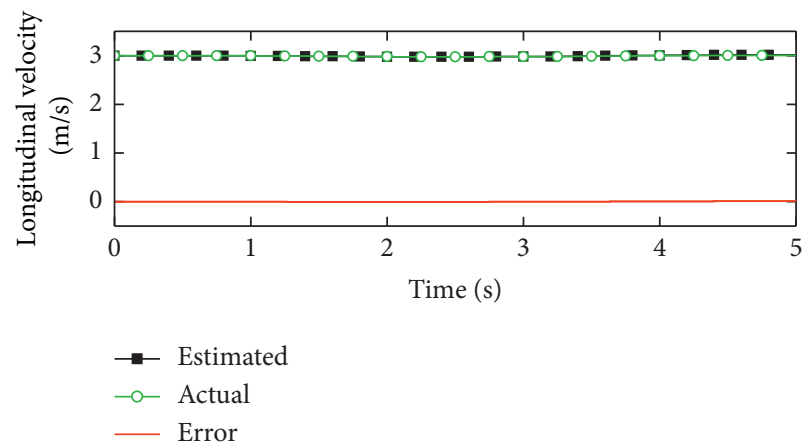

(a)

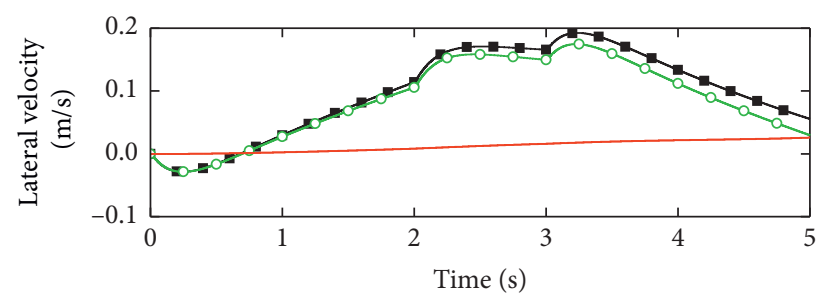

$$
\begin{aligned}
& \longrightarrow \text { Estimated } \\
& -\circ \text { Actual } \\
& \longrightarrow \text { Error }
\end{aligned}
$$

Figure 9: Estimated velocities based on the estimator C1 (estimator C1 is without yaw rate sensors). (a) Longitudinal velocity. (b) Lateral velocity.

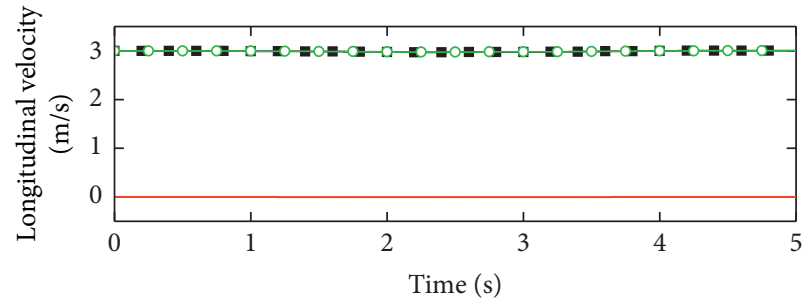

$\rightarrow-$ Estimated
$-0-$ Actual

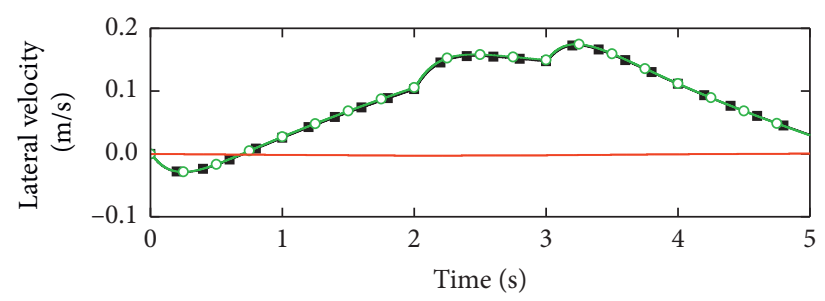
- Estimated
$-\infty$ Actual
- Error

(a)

(b)

FIGURE 10: Estimated velocities based on the estimator C2 (estimator C2 is with yaw rate sensors). (a) Longitudinal velocity. (b) Lateral velocity. 
TABLE 2: MAE and RMSE of the estimated velocities based on the estimators C1 and C2.

\begin{tabular}{lll}
\hline Estimators & MAE & RMSE \\
\hline Estimated longitudinal velocity based on the estimator C1 & 0.0136 & 0.0054 \\
Estimated lateral velocity based on the estimator C1 & 0.0260 & 0.0150 \\
Estimated longitudinal velocity based on the estimator C2 & 0.0038 & 0.0021 \\
Estimated lateral velocity based on the estimator C2 & 0.0026 & 0.0016 \\
\hline
\end{tabular}

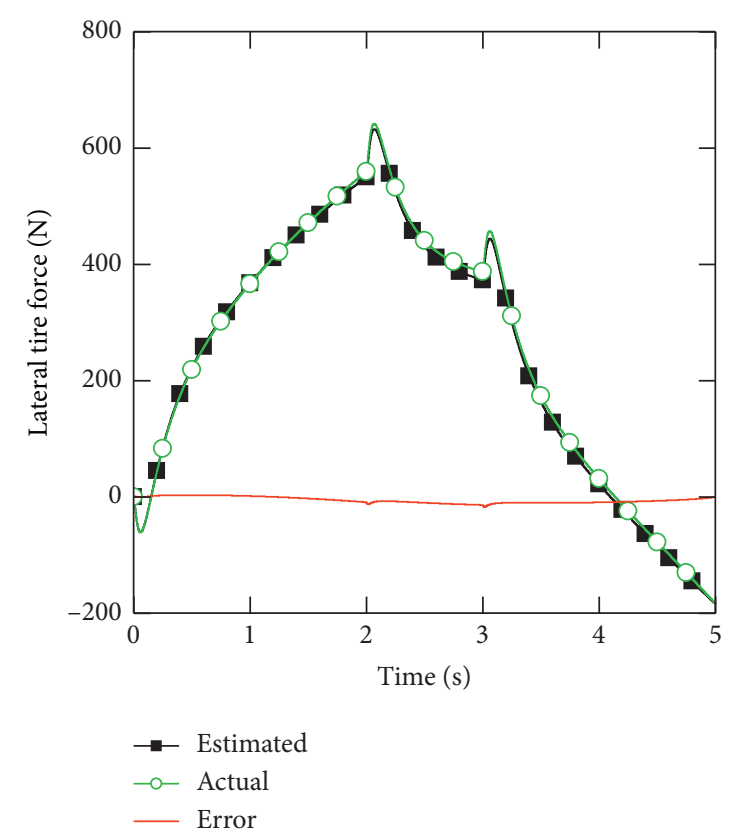

(a)

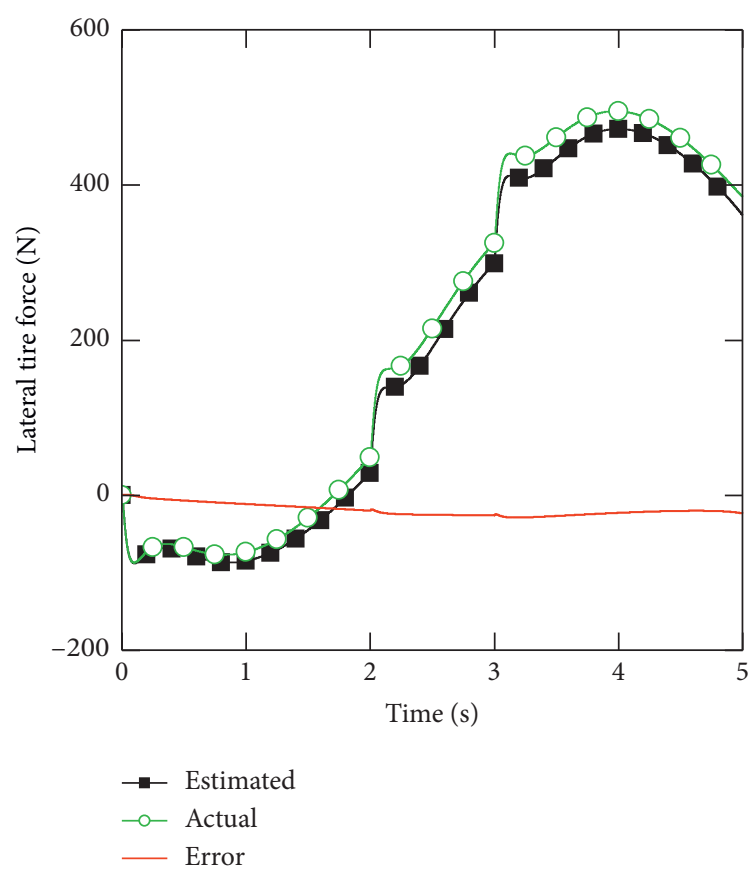

(c)

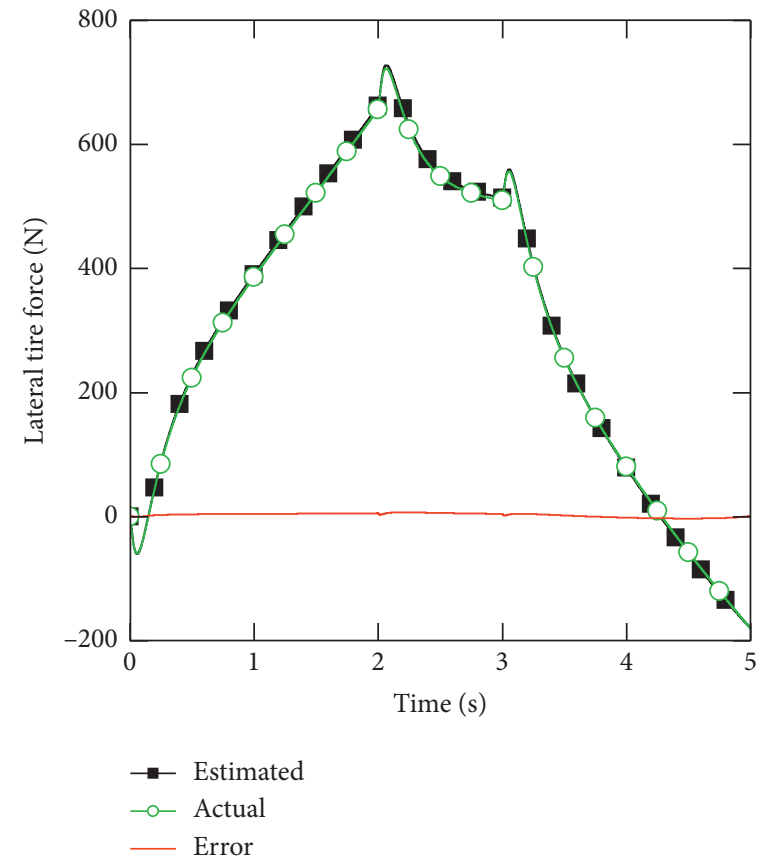

(b)

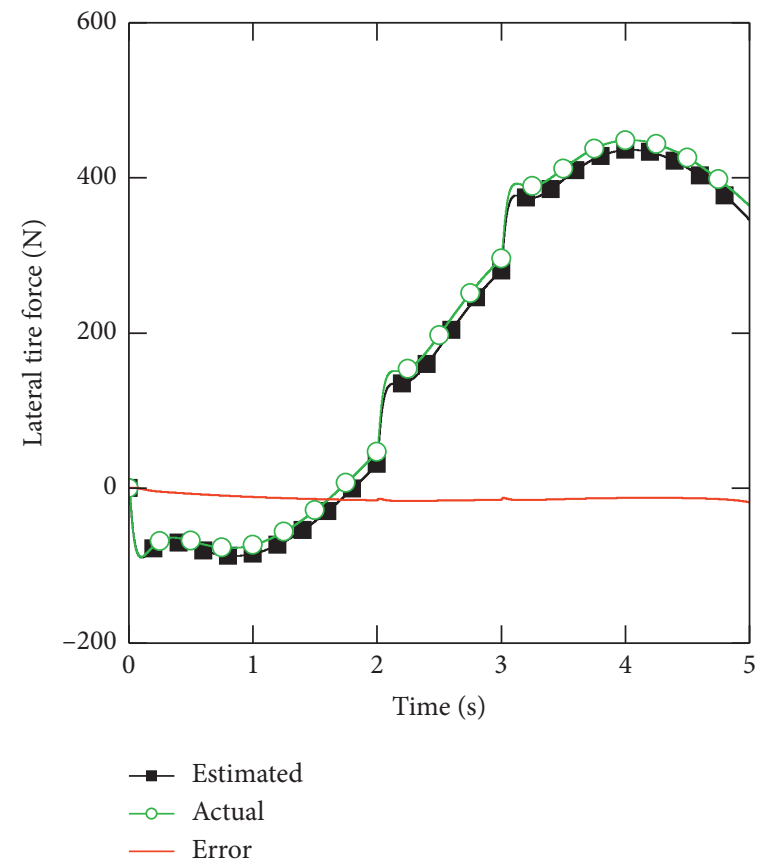

(d)

FIGURE 11: Estimated lateral tire forces based on the estimator D1 (estimator D1 is based on the transient tire model without yaw rate sensors). (a) Front left lateral tire force. (b) Front right lateral tire force. (c) Rear left lateral tire force. (d) Rear right lateral tire force. 


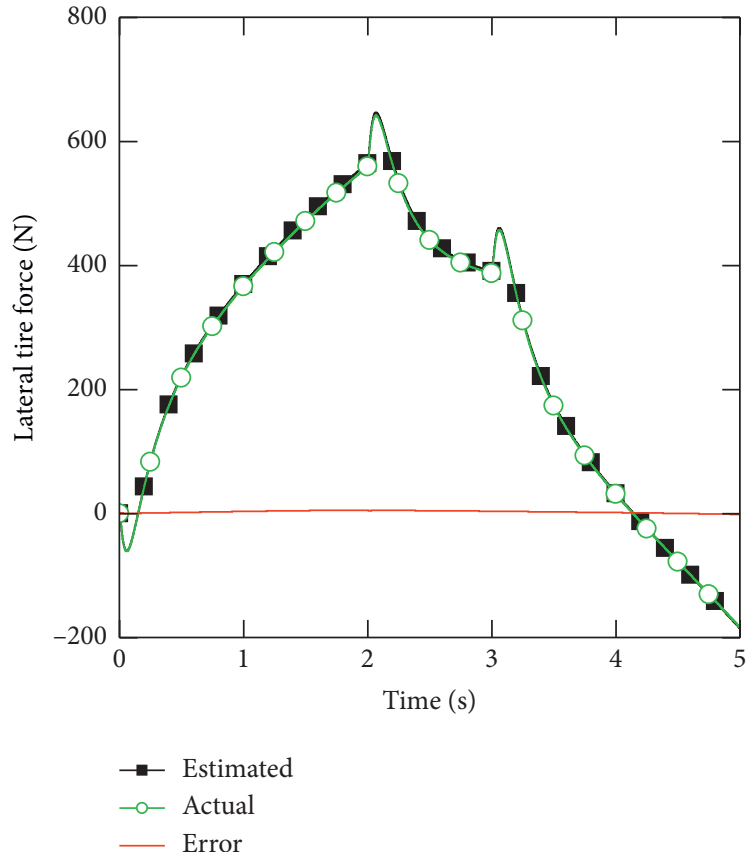

(a)

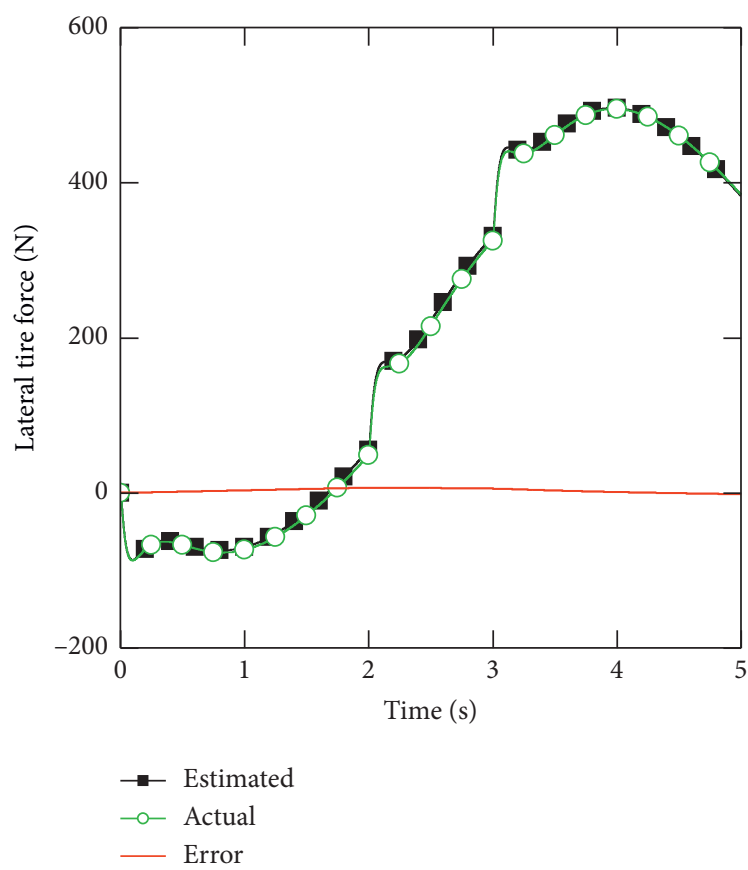

(c)

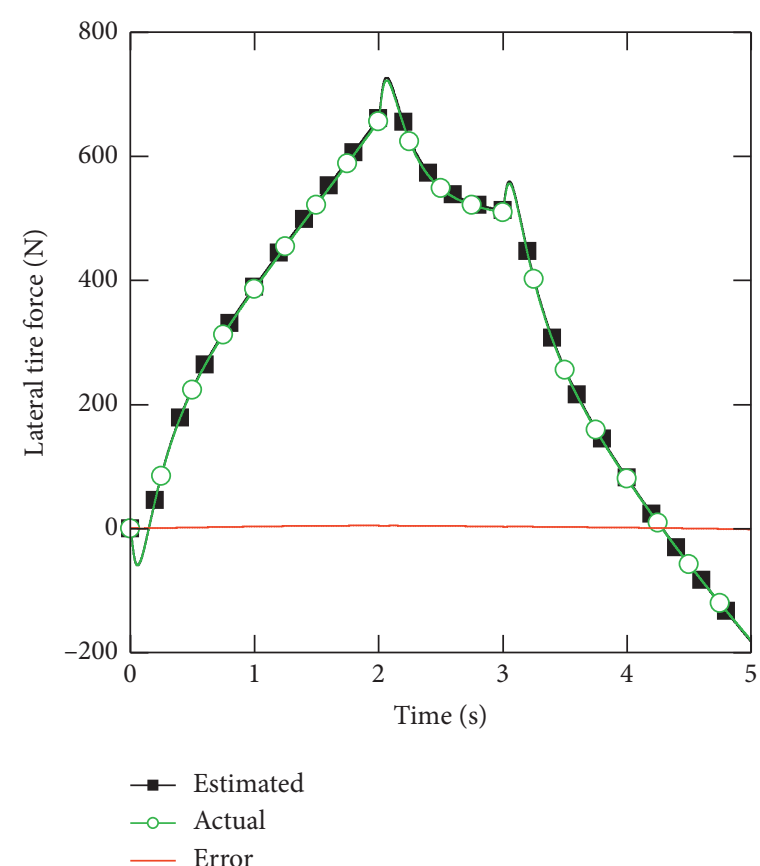

(b)

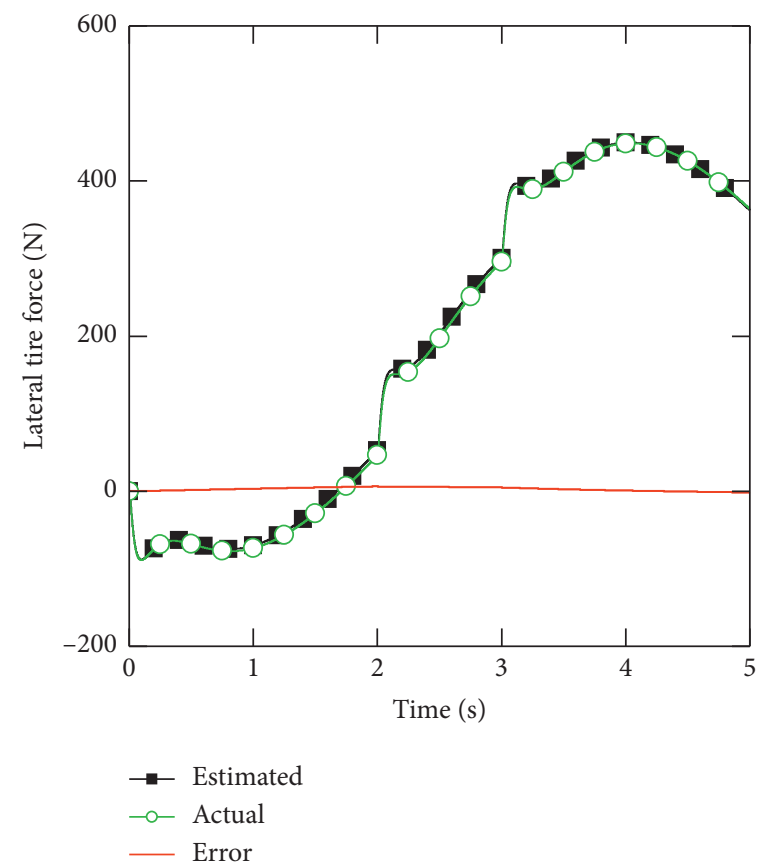

(d)

FIGURE 12: Estimated lateral tire forces based on the estimator D2 (estimator D2 is based on the transient tire model with yaw rate sensors). (a) Front left lateral tire force. (b) Front right lateral tire force. (c) Rear left lateral tire force. (d) Rear right lateral tire force.

estimated lateral tire forces based on the estimator B1 are $0.0446 \%, 0.0369 \%, 0.0223 \%$, and $0.0226 \%$, which, respectively, denote the result of the front left, front right, rear left, and rear right tires. Same as above, the EMSPEs of the estimated lateral tire forces based on the estimator B2 are $0.0446 \%, 0.0379 \%, 0.0258 \%$, and $0.0257 \%$.

Figure 9 shows the estimated velocities based on the estimator $\mathrm{C} 1$. Figure 10 shows the estimated velocities based on the estimator C2. The EMSPEs of the estimated velocities based on the estimator $\mathrm{C} 1$ are $0.0008 \%$ and $0.0629 \%$, which, respectively, denote the results of the longitudinal and lateral velocities. Same as above, the EMSPEs of the estimated velocities based on the estimator C2 are $0.0003 \%$ and $0.0066 \%$.

Table 2 summarizes the MAE and RMSE for velocities estimated by estimators $\mathrm{C} 1$ and $\mathrm{C} 2$. The results demonstrate that the estimated accuracy of the estimator $\mathrm{C} 2$ is better than that of 
TABLE 3: MAE and RMSE error of the estimated lateral tire forces based on different estimators.

\begin{tabular}{lcc}
\hline Estimators & MAE & RMSE \\
\hline Estimator B1 & $(62.85,69.67,29.10$, & $(33.85,32.71,15.4,14.2)$ \\
\hline \multirow{2}{*}{ Estimator B2 } & $(62.94,69.25,45.75$, & $(34.95,33.60,17.86$, \\
& $47.14)$ & $16.02)$ \\
\hline Estimator & $(17.50,6.673,28.87$, & $(7.831,3.902,20.30$, \\
D1 & $18.26)$ & $13.26)$ \\
\hline Estimator & $(5.193,4.556,6.778$, & $(3.190,2.877,4.085$, \\
D2 & $6.367)$ & $3.753)$ \\
\hline
\end{tabular}

the estimator $\mathrm{C} 1$. The average improvements about MAE and RMSE from the estimators $\mathrm{C} 1$ to $\mathrm{C} 2$ are $83.84 \%$ and $80.39 \%$.

Figure 11 shows the estimated lateral tire forces based on the estimator D1. Figure 12 shows the estimated lateral tire forces based on the estimator D2. The EMSPEs of the estimated lateral tire forces based on the estimator D1 are $0.0103 \%, 0.0044 \%, 0.0293 \%$, and $0.0210 \%$, which, respectively, denote the results of the front left, front right, rear left, and rear right tires. Same as above, The EMSPEs of the estimated lateral tire forces based on the estimator D2 are $0.0446 \%, 0.0042 \%, 0.0059 \%$, and $0.0060 \%$.

Table 3 summarizes the MAE and RMSE, respectively, for the front left, front right, rear left, and rear right lateral tire forces, estimated based on different estimators. The average improvements about MAE and RMSE from the estimators $\mathrm{B} 2$ to $\mathrm{B} 1$ are $-17.51 \%$ and $-6.520 \%$. The average improvements about MAE and RMSE from the estimators D2 to D1 are $67.89 \%$ and $68.97 \%$. The yaw rate sensors show no superiority based on the quasistatic tire model, but the superiority is obvious based on the transient tire model.

The average improvements about MAE and RMSE from the estimators D1 to $\mathrm{B} 1$ are $62.77 \%$ and $53.37 \%$. The average improvements about MAE and RMSE from the estimators D2 to $\mathrm{B} 2$ are $89.83 \%$ and $86.42 \%$.

\section{Conclusions}

A comparative study about how to estimate the velocities and tire forces of DDAVS is presented. Two factors are compared by multistage estimators, respectively. One is yaw rate sensors, and the other is the tire model.

Without the ESP sensors, the measurement sensor schemes need to be explored. In this paper, two kinds of multistage estimators were established: one was equipped with acceleration sensors only and the other with the additional three yaw rate sensors. For each multistage estimator, this paper compared the estimator based on the quasistatic tire model and transient tire model.

Regarding the EMSPEs, the estimate results for velocities and tire forces of the DDASV based on different multistage estimators differentiated by the yaw sensors and the tire models are all favourable to a certain extent, while the estimated accuracy of different estimators is discrepant.
(1) For the estimated velocities, the yaw rate sensors can improve the estimated accuracy.

(2) For the estimated lateral tire forces based on the quasistatic tire model, the performance of the estimator with the yaw rate sensors is not better than that of the estimator without the yaw rate sensors regarding the estimated accuracy. But the improvement is significant if it is based on the transient tire model.

(3) For the estimated lateral tire forces, the estimated accuracy based on the transient tire model is better than that based on the quasistatic tire model, whether the estimator has yaw rate sensors or not.

(4) For ameliorating the estimated accuracy of the lateral tire forces, the improvement by using the transient tire model is more effective than equipping yaw rate sensors.

The simulation in this paper is based on the conventional pavement. Furthermore, the simulation elements can be extended to off-road, which can enhance the availability of the estimator. Meanwhile, more degrees of freedom of DDASV need to be taken into consideration, like rolling movement and pitch movement.

\section{Nomenclature}

$m: \quad$ Gross mass

$R_{x j}: \quad$ Longitudinal tire resistance, $j=1,2,3,4$, represents the position as front left, front right, rear left, and rear right, respectively

$F_{x j}: \quad$ Longitudinal tire force, $j=1,2,3,4$ represents the position as front left, front right, rear left, and rear right, respectively

$F_{y j}: \quad$ Lateral tire force, $j=1,2,3,4$ represents the position as front left, front right, rear left, and rear right, respectively

$\delta$ :

$a_{x}:$ Swing angle

Longitudinal acceleration

$m_{f}: \quad$ Mass of the front part vehicle

$v_{i j}$ : Front and rear vehicle velocity, $i j=x f$ represents the longitudinal velocity of front vehicle, $i j=y f$ represents the lateral velocity of front vehicle, $i j=x r$ represents the longitudinal velocity of rear vehicle, and $i j=y r$ represents the lateral velocity of rear vehicle

$\varphi: \quad$ Yaw rate

$\varphi_{f}: \quad$ Yaw rate of the front part vehicle

$\varphi_{r}: \quad$ Yaw rate of the rear part vehicle

$m_{r}: \quad$ Mass of the rear part vehicle

$a_{y}: \quad$ Lateral acceleration

$v_{x}: \quad$ Longitudinal velocity

$v_{y}: \quad$ Lateral velocity

$l_{m f}: \quad$ Distance from articulated point to the center of front vehicle gravity

$l_{m r}: \quad$ Distance from articulated point to rear axle

$I_{z}: \quad$ Vehicle rotational inertia about $z$-axis 


\begin{tabular}{|c|c|}
\hline$B_{f}:$ & Front wheel track \\
\hline$B_{r}:$ & Rear wheel track \\
\hline$l_{f}:$ & Distance from articulated point to front axle \\
\hline$l_{r}:$ & Distance from articulated point to rear axle \\
\hline$I_{z f}:$ & $\begin{array}{l}\text { Front part vehicle rotational inertia about } z \\
\text {-axis }\end{array}$ \\
\hline$I_{z r}:$ & Rear part vehicle rotational inertia about $z$-axis \\
\hline$h:$ & Centroid height \\
\hline$\sigma_{x}:$ & $\begin{array}{l}\text { Longitudinal slip rate, } j=1,2,3,4 \text { represents } \\
\text { the position as front left, front right, rear left, } \\
\text { and rear right, respectively }\end{array}$ \\
\hline$C_{\sigma}:$ & Longitudinal tire stiffness \\
\hline$\alpha:$ & Sideslip angle \\
\hline$C_{\alpha}:$ & Lateral tire stiffness \\
\hline$\mu:$ & Friction coefficient \\
\hline$X(k)$ : & State vector \\
\hline$W(k):$ & System noise matrix \\
\hline$Z(k):$ & Measurement vector \\
\hline$V(k):$ & Measurement noise covariance matrix \\
\hline$\widehat{X}(k):$ & One-step-ahead prediction state vector \\
\hline$P(k):$ & System covariance matrix \\
\hline$x_{i}(k):$ & Sampling state vector \\
\hline$x_{i}(k / k-1):$ & $\begin{array}{l}\text { One-step-ahead prediction sampling state } \\
\text { vector }\end{array}$ \\
\hline$\widehat{X}(k / k-1):$ & $\begin{array}{l}\text { One-step-ahead prediction estimate state } \\
\text { vector }\end{array}$ \\
\hline $\begin{array}{l}P(k / k-1): \\
\zeta_{i}(k / k-1):\end{array}$ & $\begin{array}{l}\text { One-step-ahead system covariance matrix } \\
\text { One-step-ahead sampling measurement vector }\end{array}$ \\
\hline$\widehat{Z}(k / k-1):$ & $\begin{array}{l}\text { One-step-ahead prediction estimate } \\
\text { measurement vector }\end{array}$ \\
\hline$P_{Z Z}:$ & Measurement covariance matrix \\
\hline$R:$ & Measurement noise covariance matrix \\
\hline$P_{X Z}:$ & $\begin{array}{l}\text { Covariance matrix between measurement and } \\
\text { state vector }\end{array}$ \\
\hline$K(k):$ & Gain matrix \\
\hline Q: & System noise covariance matrix \\
\hline$\omega_{j}:$ & $\begin{array}{l}\text { Wheel rotate speed, } j=1,2,3,4 \text { represents the } \\
\text { position as front left, front right, rear left, and } \\
\text { rear right, respectively. }\end{array}$ \\
\hline
\end{tabular}

\section{Data Availability}

The data used to support the findings of this study are available from the corresponding author upon request.

\section{Conflicts of Interest}

The authors declare that there are no conflicts of interest regarding the publication of this paper.

\section{Acknowledgments}

This research was supported by the State Key Laboratory of Smart Manufacturing for Special Vehicles and Transmission System (grant no. GZ2018KF005) and National Natural Science Foundation of China (grant no. 51875239).

\section{References}

[1] Z. Wang, Y. Wang, L. Zhang, and M. Liu, "Vehicle stability enhancement through hierarchical control for a four-wheel-independently-actuated electric vehicle," Energies, vol. 10, no. 7, p. 947, 2017.

[2] T. Xu, Y. Shen, and W. Zhang, "In-situ steering dynamics analysis of skid steering for articulated motor-driven vehicle," SAE International Journal of Passenger Cars-Mechanical Systems, vol. 9, no. 2, pp. 903-911, 2016.

[3] X. Zhang and D. Göhlich, "Improvement in the vehicle stability of distributed-drive electric vehicles based on integrated model-matching control," Proceedings of the Institution of Mechanical Engineers, Part D: Journal of Automobile Engineering, vol. 232, no. 3, pp. 341-356, 2018.

[4] K. B. Singh, M. A. Arat, and S. Taheri, "Literature review and fundamental approaches for vehicle and tire state estimation," Vehicle System Dynamics, vol. 57, no. 11, pp. 1643-1665, 2019.

[5] M. Acosta, S. Kanarachos, and M. Blundell, "Virtual tyre force sensors: an overview of tyre model-based and tyre model-less state estimation techniques," Proceedings of the Institution of Mechanical Engineers, Part D: Journal of Automobile Engineering, vol. 232, no. 14, pp. 1883-1930, 2018.

[6] C. Huang, F. Lei, X. Han, and Z. Zhang, "An improved adaptive unscented Kalman filter for estimating the states of in-wheel-motored electric vehicle," International Journal of Adaptive Control and Signal Processing, vol. 33, no. 11, pp. 1676-1694, 2019.

[7] X. Zhang, D. Göhlich, and C. Fu, "Comparative study of two dynamics-model-based estimation algorithms for distributed drive electric vehicles," Applied Sciences, vol. 7, no. 9, p. 898, 2017.

[8] S. Hou, W. Xu, and G. Liu, "Design of an interacting multiple model-cubature Kalman filter approach for vehicle sideslip angle and tire forces estimation," Mathematical Problems in Engineering, vol. 2019, Article ID 6087450, 13 pages, 2019.

[9] W. Zhang, N. Ding, G. Yu, and W. Zhou, "Virtual sensors design in vehicle sideslip angle and velocity of the centre of gravity estimation," in Proceedings of the 2009 9th International Conference on Electronic Measurement \& Instruments, pp. 3652-3656, Beijing, China, August 2009.

[10] M. Acosta and S. Kanarachos, "Tire lateral force estimation and grip potential identification using neural networks, extended Kalman filter, and recursive least squares," Neural Computing and Applications, vol. 30, no. 11, pp. 3445-3465, 2018.

[11] G. Morrison and D. Cebon, "Sideslip estimation for articulated heavy vehicles at the limits of adhesion," Vehicle System Dynamics, vol. 54, no. 11, pp. 1601-1628, 2016.

[12] H. Ahmadi Jeyed and A. Ghaffari, "Nonlinear estimator design based on extended Kalman filter approach for state estimation of articulated heavy vehicle," Proceedings of the Institution of Mechanical Engineers, Part K: Journal of MultiBody Dynamics, vol. 233, no. 2, pp. 254-265, 2019.

[13] B. Xu, F. Mu, G. Shi, W. Ji, and H. Zhu, "State estimation of permanent magnet synchronous motor using improved square root UKF," Energies, vol. 9, no. 7, p. 489, 2016.

[14] J. Rong, S. Song, Z. Dang, H. Shi, and Y. Cao, "Rail track irregularity detection method based on computer vision and gesture analysis," International Journal of Online Engineering (IJOE), vol. 12, no. 12, p. 55, 2016.

[15] Z. Li, H. Zhang, Q. Zhou, and H. Che, "An adaptive low-cost INS/GNSS tightly-coupled integration architecture based on 
redundant measurement noise covariance estimation," Sensors, vol. 17, no. 9, p. 2032, 2017.

[16] X. U. Lun-Hui and W. Xiang-Xue, "Application of SVD-based optimized robust UKF algorithm to estimation of short-term traffic flow state," Journal of South China University of Technology, vol. 44, no. 12, pp. 44-52, 2016.

[17] K. Lu, "Strong track schmidt filter and its application to speed sensorless control of induction motor: strong track schmidt filter and its application to speed sensorless control of induction motor," Acta Automatica Sinica, vol. 34, no. 9, pp. 1076-1082, 2009.

[18] L. Wang, L. Wu, Y. Guan, and G. Wang, "Online sensor fault detection based on an improved strong tracking filter," Sensors, vol. 15, no. 2, pp. 4578-4591, 2015.

[19] X. Ning, Z. Li, W. Wu, Y. Yang, J. Fang, and G. Liu, "Recursive adaptive filter using current innovation for celestial navigation during the Mars approach phase," Science China Information Sciences, vol. 60, no. 3, Article ID 032205, 2017. 